\title{
Characteristics of diamond turned NiP smoothed with ion beam planarization technique
}

\author{
Yaguo $\mathrm{Li}^{1,2}$, Hideo Takino ${ }^{3}$ and Frank Frost ${ }^{1 *}$ (D)
}

\begin{abstract}
Background: Diamond turning is widely used in machining metals and semiconductors but the turning marks are incurred on machined components due to the mechanics of the technology. The marks are generally harmful to the systems comprising of the machined components. Therefore, the capability of ion beam planarization (IBP) to reduce turning marks of diamond turned metal surfaces was investigated using NiP as an example.

Methods: The turning marks and thereby roughness was reduced by IBP with respect to different spatial wavelengths and amplitudes of turning marks. Different thickness of coating resist was also examined in order to find out the potential effects of resist thickness on the reduction of turning marks and roughness. Additionally, the effect of multiple planarization steps was also analyzed.

Results: The spatial wavelength and depth of turning marks have only minor impact on the degree of surface roughness reduction. Thicker coating tends to achieve smoother surface after coating turned NiP while ion beam etching can keep surface roughness almost unchanged in our experiments. The surface roughness of diamond turned NiP drops exponentially with processing steps under experimented conditions. Using up to five IBP steps, the surface roughness can be reduced up to one order of magnitude (from $R_{q} \sim 6.5 \mathrm{~nm}$ to $R_{q} \sim 0.7 \mathrm{~nm}$ ).

Conclusions: IBP technique performs very well in reducing turning marks on diamond turned NiP surfaces. The surface roughness can be further improved by optimizing the properties of planarizing resist layer and coating processes to enhance the IBP technique as a final surface finishing technology.
\end{abstract}

Keywords: Ion beam planarization, Diamond turned optics; turning marks, Surface roughness, Spray/spin coating

\section{Background}

In optical fabrication, single-crystal diamond turning is effectively used to cut metal surfaces to obtain smooth and highly accurate surfaces relevant for different types of optics. The final surface roughness is mainly caused by the tool marks forming along the tool paths, which is sometimes referred to as mid spatial frequency $(1 \mu \mathrm{m} \sim$ $1 \mathrm{~mm}$ ) [1]. The turning marks will increase the scattering and deteriorate the performance of optics, in particular for those used at very short wavelength like extreme ultraviolet lithography (EUVL) and X-ray optics. Hence the turning marks must be eliminated or mitigated in the

\footnotetext{
* Correspondence: frank.frost@iom-leipzig.de

'Physical Department, Leibniz Institute of Surface Modification, Permoserstr.

15, 04318 Leipzig, Germany

Full list of author information is available at the end of the article
}

subsequent steps. Ion beam machining is a much deterministic process for final modification to surface of optics. However, on some occasions, ion beam machining alone is incapable of improving surface roughness. Hence ion beam planarization (IBP) was proposed that combines coating and ion beam machining to smooth the surface of optics [2].Within the IBP technique, smoothing results from removal of a planarizing sacrificial layer. A layer of coating (sacrificial layer), e.g. photoresist, is first applied onto the surface of optics to be smoothed. The surface gets much smooth after being coated and subsequently the sacrificial layer is removed by ion beam machining at the planarization angle (i.e., at an angle where the removal rates of the resist and the underlying substrate are nearly identical) [2-6]. Thus, the smooth surface of the sacrificial layer is transferred to the substrate [5]. This way, the 
workpiece can be readily planarized which it is otherwise difficult to planarize directly with ion beam. Frost et al. have successfully smoothed diamond turned copper and electroless plated NiP surface using IBP technique [4-6]. Although it has been shown that IBP can smooth diamond turned surfaces, many factors affect the results of smoothing effects of turning marks. Thereby the smoothing effectiveness of IBP technique on the diamond turned surface was investigated in detail. Our previous results have shown that the surface roughness can be reduced to $\sim 1 / 3$ of roughness of diamond turned surface, regardless of the spatial wavelength and depth of turning marks [7]. Long time processing with ion beam has marginal effect on the surface roughness of diamond turned surface once the resist is removed. In this paper, the potential influence of thickness of resist and multi-step processing using IBP on surface roughness of diamond turned surface was experimentally studied. It turns out that thicker resist will yield smoother surface after coating diamond turned NiP and the roughness decreases exponentially with repeating IBP processes (from $\mathrm{R}_{\mathrm{q}} \sim 6.5 \mathrm{~nm}$ to $\mathrm{R}_{\mathrm{q}} \sim 0.7 \mathrm{~nm}$ after repeating IBP processing 5 times).

\section{Methods}

For the evaluation of the ion beam planarization process six electroless plated NiP samples were diamond-turned to generate different spatial frequencies and depths of turning marks by varying the feed rate, the cut depth, and the tip radius of the tool (Table 1). The samples were then spin-coated with photoresist AZ1505 and then dried with an electric heater. For the investigations presented here only samples A and D have been used. It is expected that the results are applicable for all samples. Resists of different thickness $d$ were coated to test the possible influence of coating thickness on the surface roughness of coated $\mathrm{NiP}$ and final roughness of ion beam etched NiP. In the experiments of repeating steps, the thickness was kept roughly the same, $d \sim 300 \mathrm{~nm}$ to find out the effectiveness of IBP on reducing roughness. Samples were mounted on a water-cooled substrate stage. All experiments were normally at room temperature,

Table 1 Properties of samples after diamond turning (see Ref. [7]). For the investigations presented here only samples A and D have been used

\begin{tabular}{llll}
\hline Sample & $\begin{array}{l}\text { Spatial } \\
\text { wavelength }\end{array}$ & $\begin{array}{l}\text { Depth of turning } \\
\text { marks }\end{array}$ & $\begin{array}{l}\text { Surface } \\
\text { roughness }\end{array}$ \\
\hline A & $1.5 \mu \mathrm{m}$ & $20 \mathrm{~nm}$ & $6.49 \mathrm{~nm}$ \\
B & $6 \mu \mathrm{m}$ & $60 \mathrm{~nm}$ & $19.91 \mathrm{~nm}$ \\
C & $3.5 \mu \mathrm{m}$ & $20 \mathrm{~nm}$ & $7.63 \mathrm{~nm}$ \\
D & $6 \mu \mathrm{m}$ & $20 \mathrm{~nm}$ & $6.51 \mathrm{~nm}$ \\
E & $6 \mu \mathrm{m}$ & $10 \mathrm{~nm}$ & $4.99 \mathrm{~nm}$ \\
F & $25 \mu \mathrm{m}$ & $60 \mathrm{~nm}$ & $16.4 \mathrm{~nm}$ \\
\hline
\end{tabular}

additionally, the sample stage was rotated around the sample surface normal. The ion source is two-grid Kaufmantype and Ar was used as a process. The grids are made of graphite with a grid aperture of $180 \mathrm{~mm}$, which corresponds with FWHM of the beam. The beam voltage and the beam current were set to $700 \mathrm{~V}$ and $70 \mathrm{~mA}$, respectively. The incident angle of the ion beam, with respect to the sample normal, was $35^{\circ}$ based on our experimental results of the etching rate of photoresist and $\mathrm{NiP}$ (the similar etching rates can be achieved at this angle). A typical surface before and after one IPB step is shown in Fig. 1.

The etching time depends on the thickness of photoresist. Typical etching times were $30 \mathrm{~min}$ to remove the resist. Additional $5 \mathrm{~min}$ ion beam etching continued after the photoresist was etched away, as detailed in Ref. [7].

Surface roughness was evaluated with an atomic force microscope (Brucker Dimension Icon, Germany) before and after spin coating and after ion beam planarization; the thickness of resist was measured with a thin film analyzing system (Mikropack NanoCalc 2000, Germany) whenever it is necessary. In most cases, the AFM scan size was $80 \mu \mathrm{m} \times 80 \mu \mathrm{m}$. The AFM raw data were processed by a line-wise levelling (flatten) using a polynomial of 1st order in order to realize sample tilt/offset compensation and to remove noise along the slow scan direction. The planarization angle was $\sim 35^{\circ}$ where the etching rates of resist and NiP are approximately the same, $10 \sim$ $12 \mathrm{~nm} / \mathrm{min}$. The etching time is set to the time needed to etch away resist (depending on the resist thickness) plus additional 5 min unless otherwise specified. The detailed procedure is presented in Ref. [7].

\section{Results and discussion}

The IBP process is able reduce surface roughness to $30 \% \sim 40 \%$ of initial diamond turned surfaces of NiP after a single step for all samples, irrespective of spatial wavelength (from $1.5 \mu \mathrm{m}$ up to $25 \mu \mathrm{m}$ ) and depth of diamond turning marks (amplitudes from $10 \mathrm{~nm}$ to $60 \mathrm{~nm}$ ), which has been presented previously [7]. The degree of roughness reduction is mainly limited by the planarizing behavior of the resist (see Ref. [7] and later discussion in section 3.2).

In general, the driving force for surface levelling is a combination of surface tension and gravity, which is opposed by the viscosity of the liquid (resist) [8]. Based on Ref. [8] the exponential decay time $T$ for each Fourier components of surface roughness depends on material properties (surface tension $\gamma$, viscosity $\eta$, resist thickness $d$ ) and the respective spatial frequency $f$ (or spatial wavelength $\lambda$ ). For very thick layers of resist, i. e., $d>\lambda / 2 \pi$, the decay time is independent on resist thickness. In contrast, for very thin layers $(d<<\lambda / 2 \pi)$ the decay time is proportional to $1 / d^{3}$. If one looks at the spatial frequency range covered by the AFM measurements and 

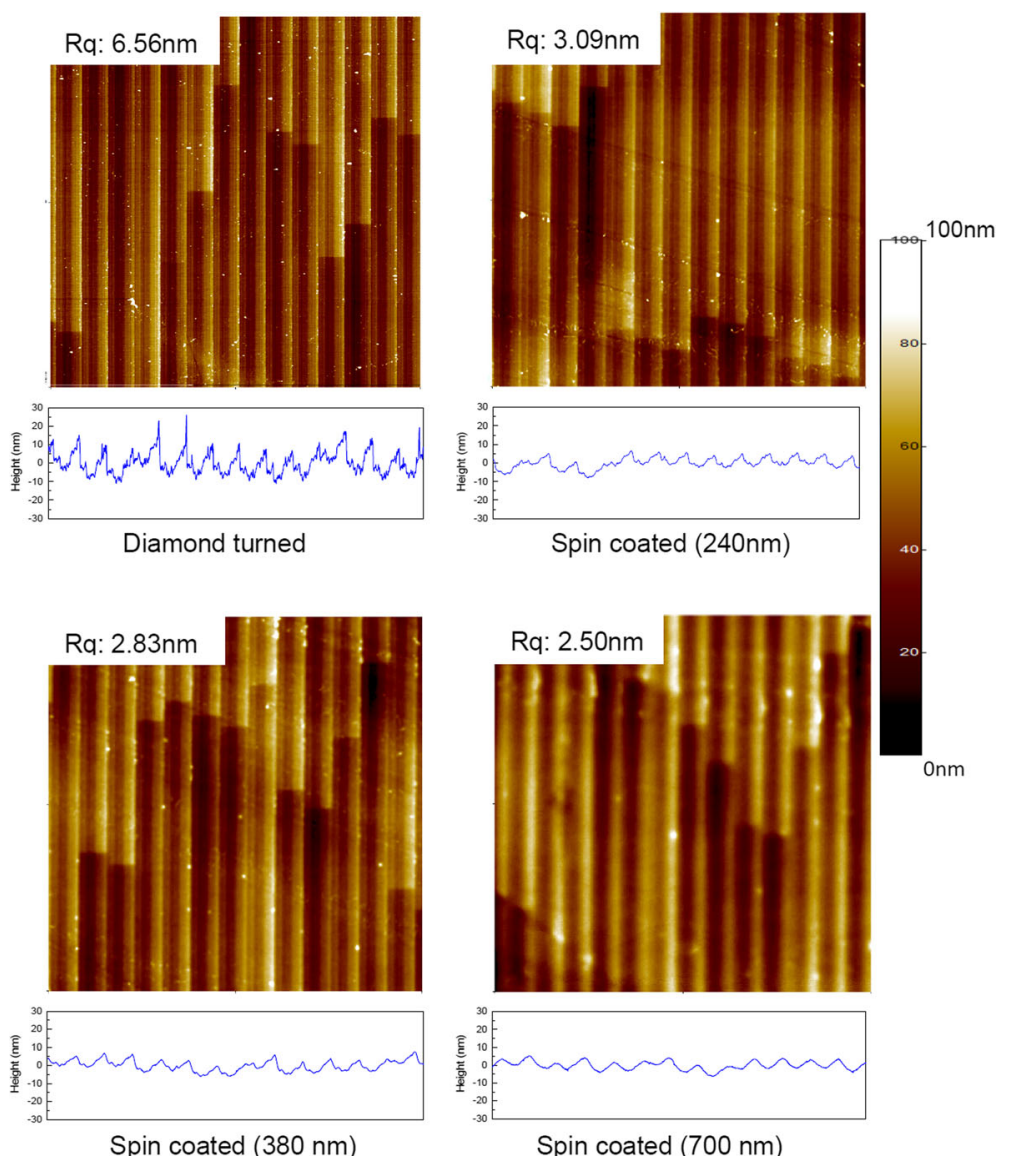

Fig. 1 Surface morphology and roughness (sample D) after coating with various thickness of photoresist (figures in parentheses indicate surface roughness). The image size is $80 \mu \mathrm{m} \times 80 \mu \mathrm{m}$ for spin-coated samples and $85 \mu \mathrm{m} \times 85 \mu \mathrm{m}$ for diamond turned one

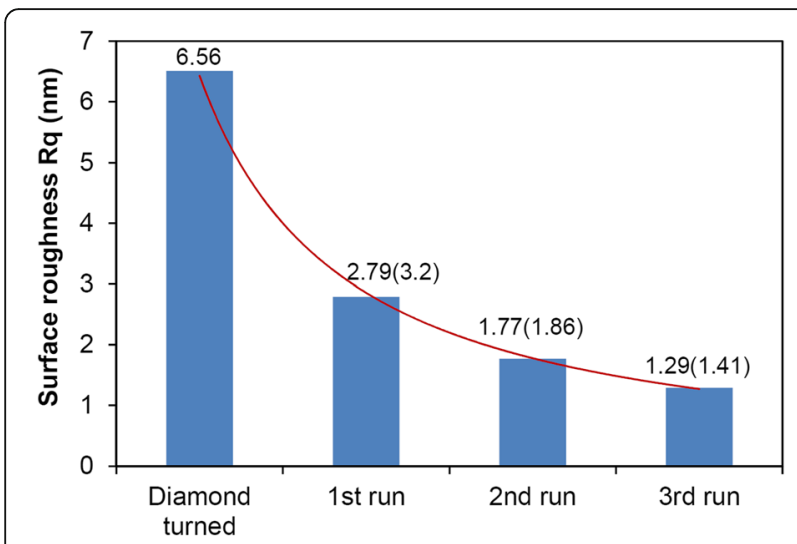

Fig. 2 Surface roughness (1.29 nm) after repeating 3 times of IBP processes on sample $\mathrm{D}$. The surface roughness of NiP is found to decrease exponentially. The numbers in parentheses are the surface roughness (RMS) values after spin-coating but prior to ion beam etching in each step. The numbers outside the parentheses are roughness after each IBP (i.e. after ion beam etching) step

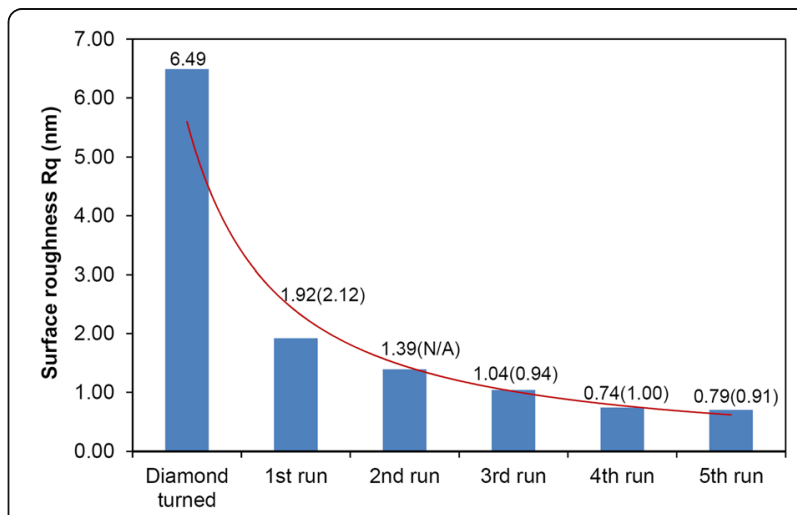

Fig. 3 Surface roughness (RMS $0.70 \mathrm{~nm}$ ) after repeating 5 times of IBP processes on sample A. The surface roughness of NiP is found to decrease exponentially. The numbers in parentheses are the surface roughness (RMS) values after spin-coating but before ion beam etching in each step. The numbers outside the parentheses are roughness after each IBP (i.e. after ion beam etching) step. N/A indicates the unavailable case 


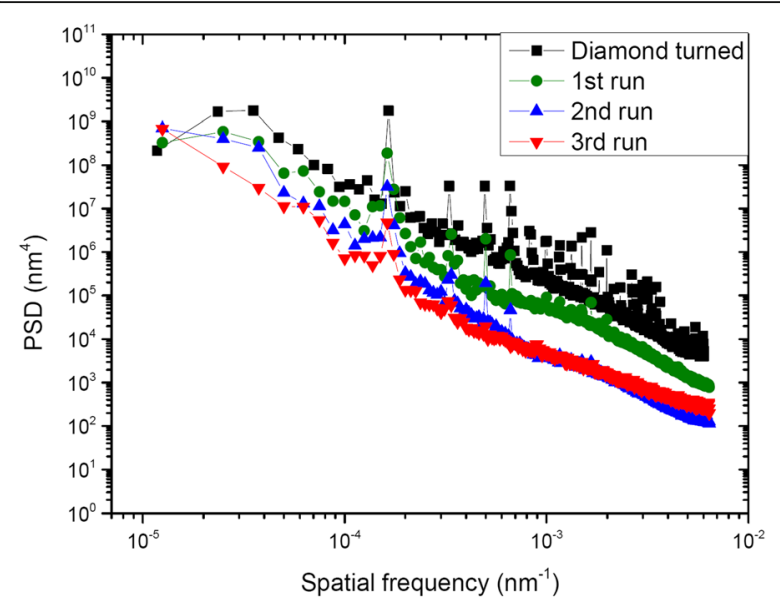

Fig. 4 PSD curves after each IBP process on sample D. The PSD plot shows that peaks at some spatial frequencies diminish with the repetition of IBP processes

under consideration of typical resist thicknesses, it can be seen that the transition range between both levelling regimes is covered within the experiments. Consequently it is not clear how the levelling changes with resist thickness. Therefore former investigations [7] were extended to test experimentally the potential effect of the thickness of coated resist and repeated coating/etching steps.

\section{Roughness of different resist thickness}

Various resist of thickness $(240 \mathrm{~nm}, 380 \mathrm{~nm}, 700 \mathrm{~nm})$ were spin coated on diamond turned NiP sample D. The surface roughness of diamond turned surface is $R_{q} 6.56 \mathrm{~nm}$ (RMS). After coating, the roughness drops down to $3.09 \mathrm{~nm}, 2.83 \mathrm{~nm}$ and $2.50 \mathrm{~nm}$, respectively (Fig. 1). The surface roughness decreases slightly with the increase in thickness of resist, which is readily understandable according the discussion above. Although thicker resist is marginally superior to thinner ones in terms of surface roughness, the etching time to remove resist will significantly increase. For example, coating $700 \mathrm{~nm}$ resist will decrease surface roughness from $\sim 3.10 \mathrm{~nm}$ to $2.50 \mathrm{~nm}$ compared to $240 \mathrm{~nm}$ resist, $19 \%$ reduction in surface roughness; however, the etching time to remove resist will increase from $\sim 22$ min to $\sim 64 \mathrm{~min}, 191 \%$ increase in etching time. Coating thicker resist can reduce surface roughness indeed, though it is not an advisable way to reduce surface roughness by virtue of making resist thicker in that it is not efficient.

\section{Repeating coating/etching (IBP) processes}

It has been shown that IBP is able to decrease the surface roughness of diamond turned NiP surface to the $~ 30 \%$ of the initial surfaces after one step. Here we repeated IBP processes to see if the roughness could further decrease by re-applying the IBP processes (coating/etching) to smoothed surface.

Sample D and A was selected in our experiments. The resist thickness was controlled as the same as possible in each step $(\sim 300 \mathrm{~nm})$. The results for sample D and A are plotted in Figs. 2 and 3, respectively. After first IBP process, the roughness for sample D and A was reduced from $6.56 \mathrm{~nm}$ and $6.49 \mathrm{~nm}$ to $2.79 \mathrm{~nm}$ and $1.92 \mathrm{~nm}$, respectively, $30 \% \sim 40 \%$ reduction rate. The surface roughness after coating for sample A $(2.12 \mathrm{~nm})$ is smaller than that of sample D (3.2 nm), so after ion beam etching the roughness of sample $\mathrm{A}$ is better than sample $\mathrm{D}$, which is attributed to different spatial wavelength of D $(6 \mu \mathrm{m})$ and A $(1.5 \mu \mathrm{m})$. The surface roughness after IBP continues decreasing as repeating IBP process, but the reduction rate gradually decreases. After repeating IBP process several times, it is found that both the sample D
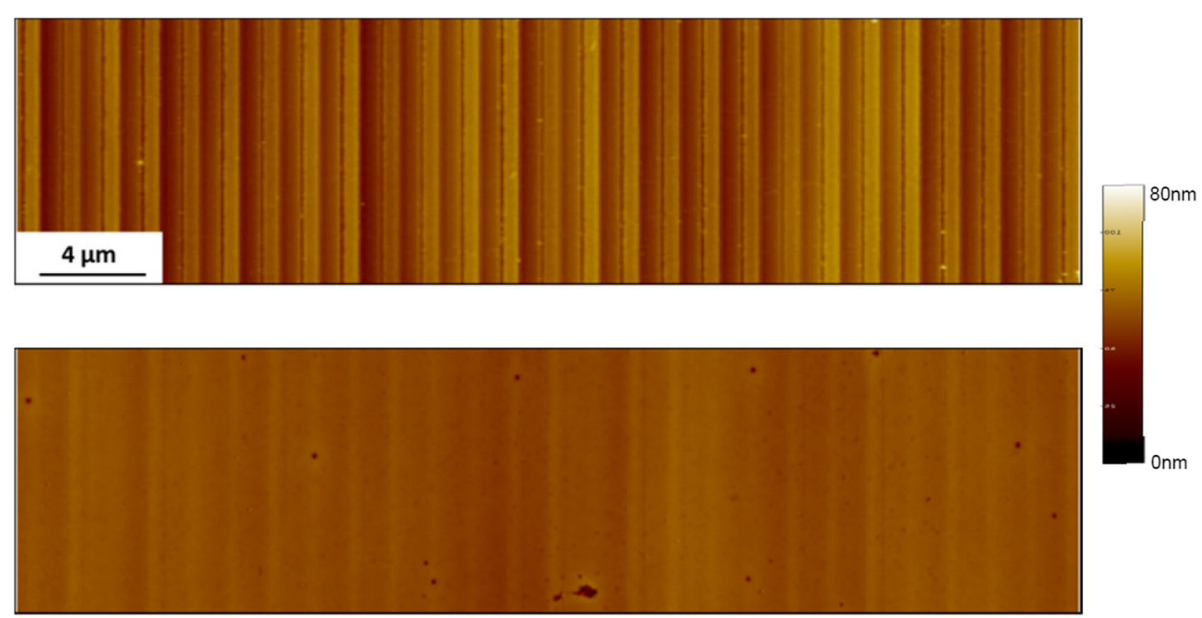

Fig. 5 AFM images $(40 \mu \mathrm{m} \times 10 \mu \mathrm{m})$ of a diamond turned surface before and after one IBP run on sample A. The amplitudes of the tool marks were reduced from $20 \mathrm{~nm}$ to $2.5-4 \mathrm{~nm}$ (height scale $80 \mathrm{~nm}$ ) 
and A behaves similarly and surface roughness drops exponentially (Figs. 2 and 3). The surface of sample D $(1.29 \mathrm{~nm})$ has been smoothed to $20 \%$ of initial diamond turned surface after 3 times IBP processes and the surface roughness of sample A $(0.70 \mathrm{~nm})$ has dropped to $10 \%$ of initial surface roughness after 5 times IBP processing. Based on Figs. 2 and 3 it is once again clarified that the roughness reduction is mainly controlled by the resist coating.

The mid spatial frequency is also able to be mitigated by means of multiple IBP processes. Shown in Fig. 4 are the PSD curves of sample A after each IBP step. It is clear that the intensity of the peak located at spatial frequency $1.7 \times 10^{-4} \mathrm{~nm}^{-1}$ abates with increasing the repetition number of IBP processes.

As seen from Fig. 5, small depressions evolve during the IBP process. These are caused by particles agglomerates in the photo resist, which are caused by aging effect. For optimized resist formulations, e.g. with respect to viscosity, further improvement of the IBP process can be expected.

\section{Conclusions}

The paper focuses on the effect of film thickness and repeating IBP processes on surface roughness reduction of diamond turned NiP. The results indicate that surface roughness of diamond-turned $\mathrm{NiP}$ can be significantly reduced by combined process of resist coating and ion beam etching (IBP process). The surface roughness drops to $30 \% \sim 40 \%$ of initial diamond turned surface after a single ion beam planarization step, irrespective of spatial wavelength and depth of diamond turning marks on NiP surfaces. Multiple IBP steps allow a further reduction of roughness to one-tenth of the initial value and improve the PSD characteristics and significantly mitigate the turning marks on the surface of diamond turned NiP.. Therefore, ion beam planarization (IBP) can be used as a final processing step to reduce the final surface roughness of diamond surfaces for a wide range of spatial wavelength and depth of tool marks. There is further potential to optimize the IBP technology, if the resist coating processes as well as the resist properties can be amended.

\footnotetext{
Abbreviations

IBP: ion beam planarization; EUVL: extreme ultraviolet lithography: AFM: atomic force microscope; RMS: root mean square; $R_{q}$ : root mean square surface roughness
}

\section{Acknowledgements}

The assistance in this work from Mr. Toni Liebeskind and Mrs. Katrin Ohndorf of the IOM is appreciated.

\section{Funding}

YL gratefully acknowledges the funding of the Deutscher Akademischer Austauschdienst (DAAD No. 57191174) and the National Natural Science Foundation of China (NSFC No. 51505444).
Availability of data and materials

All datasets on which the conclusions of the manuscript rely are included in the manuscript.

\begin{abstract}
Authors' contributions
YL conducted the experiments on IBP and engaged in writing, HT was responsible for the preparation of the diamond turned NiP samples and provided expert advice, FF involved in the experiments and writing the manuscript and coordinated the work. All the authors have read and approved the final manuscript.
\end{abstract}

Ethics approval and consent to participate

Not applicable.

Consent for publication

Not applicable.

\section{Competing interests}

The authors declare that they have no competing interests.

\section{Publisher's Note}

Springer Nature remains neutral with regard to jurisdictional claims in published maps and institutional affiliations.

\section{Author details}

${ }^{1}$ Physical Department, Leibniz Institute of Surface Modification, Permoserstr. 15, 04318 Leipzig, Germany. ${ }^{2}$ Fine Optical Engineering Research Centre, Chengdu 610041, China. ${ }^{3}$ Department of Mechanical Engineering, Faculty of Engineering, Chiba Institute of Technology, 2-17-1 Tsudanuma, Narashino, Chiba 275-0016, Japan.

Received: 14 June 2017 Accepted: 5 October 2017

Published online: 13 October 2017

References

1. Takino, H, Kanaoka, M, Nomura, K: "Ultraprecision machining of optical surfaces," 2011. JSPE Spring Meeting, Tokyo (2011)

2. Johnson, L, Ingersoll, $K$ : Ion polishing with the aid of a planarizing film. Appl. Opt. 22, 1165-1167 (1983)

3. Johnson, LF, Ingersoll, KA, Kahng, D: Planarization of patterned surfaces by ion beam erosion. Appl. Phys. Lett. 40, 636-638 (1982)

4. Frost, F, Takino, H, Fechner, R, Schindler, A, Ohi, N, Nomura, K: Smoothing of diamond-turned copper surfaces using ion beams with aid of planarizing film. Jpn. J. Appl. Phys. 46, 6071-6073 (2007)

5. Frost, F, Fechner, R, Ziberi, B, Völlner, J, Flamm, D, Schindler, A: Large area smoothing of surfaces by ion bombardment: fundamentals and applications. J. Phys. Cond. Mat. 21, 224026 (2009)

6. F. Frost, H. Takino, R. Fechner, A. Schindler, N. Ohi, and K. Nomura, "Subnanometer smoothing of diamond-turned metal surfaces using ion beams," in Towards Synthesis of Micro-/Nano-systems (Springer London), The 11th International Conference on Precision Engineering (ICPE),August 16-18, 2006, Tokyo, Japan, pp. 239-242

7. Li, Y, Takino, H, Frost, F: Ion beam planarization of diamond turned surfaces with various roughness profiles. Opt. Express. 25, 7828-7838 (2017)

8. Ochard, SE: On surface levelling in viscous liquids and gels. Appl. Sci. Res. 11A, 451-464 (1962) 